\title{
Comparison of DIC and LDV for practical vibration and modal measurements
}

Phillip L. Reu, Daniel Rohe, and Laura Jacobs

\begin{abstract}
We compare laser Doppler vibrometry (LDV) and digital image correlation (DIC) for use in full-field vibration and modal testing. This was done using a simultaneously measured 3D displacement field on a flat 7-inch corner-supported metal plate using pseudorandom excitation via a shaker. We complete a detailed comparison between the techniques and discuss the pros and cons of each. The results show that either technique can be used for quantifying the modal information with the LDV providing better out-of-plane displacement resolution and equivalent in-plane resolution. The strain calculation is considered better in the DIC approach due to the direct tie to the surface displacements. While the LDV does not lose its place as the gold standard for modal testing, DIC has introduced a new and competitive approach that will have significant advantages in certain testing regimes.
\end{abstract}

\section{Introduction}

To efficiently and effectively evaluate structures and mechanical systems, a significant amount of effort has been expended to develop testing and computational tools. Some of these tools are utilized for the correlation and updating of computational models for structural dynamic predictions. The severe mismatch between the relatively few measured degrees of freedom for the test data compared to those in finite element modeling has been a roadblock to the efficient and effective correlation and updating of these computational models (one example being in the research of mechanical joints). Vibration testing is becoming increasingly complex, especially as the practice is moving towards simultaneous excitation of multiple axes with six degree-of-freedom shakers. Therefore, being able to characterize the stress states from these complex inputs is critical. The vibration testing industry is also increasingly performing tests to failure, and it is important to capture the strains that are leading to these failures. It is also important to capture full-field measurements to be able to capture complex shape information to correlate with uncertain regions of computational models with large degrees of freedom, and characterize the behavior of structures at failure. The need for full-field diagnostics requires the ability to measure the response of a large number of points without significantly modifying the structural response.

Traditional methods for collecting experimental structural dynamics measurements involve using individual sensors, which provide measurements at discrete points by contacting the device under test (accelerometers and strain gages for example). Non-contacting techniques, such as scanning laser Doppler vibrometry (SLDV) have become well established in the modal testing community, while other 
optical techniques, such as digital image correlation (DIC), are relatively new in structural dynamics. Recent studies [1-7] have investigated the possibility of using DIC in vibration applications. All of the studies found the technique to be a viable option to generate full-field data. Helfrick et. al [2] determined that the data collected using DIC provided useful empirical data for correlation with finite element models. Niezrecki et. al [3] showed that it was possible to measure all of the points on the structure simultaneously using DIC, but only one mode at a time.

Recent studies $[1,3,8-10]$ compared the use of SLDV to determine mode shapes and generate data for model correlation with accelerometers and DIC. These studies all found that the main difference between the SLDV and DIC is that the SLDV sequentially measures all of the points on the structure, whereas DIC measures all of the points simultaneously. The sequential measurement of the points, as will be discussed in this paper, requires longer test times to capture all of the data. As with any measurement, they determined that DIC was only viable if the displacements of the modes were above the noise floor, however as shown in this paper - the noise floor of both DIC and SLDV can be in the nanometer range. The studies found that the main drawback to the use of DIC and SLDV was the large amount of data generated. The limiting factor to the efficacy is the computing power and memory available. Ehrhardt et. al [10] discovered that DIC and SLDV techniques were able to collect data to expose inconsistencies in the dynamic behavior of structures and provide insight to modeling and predicting dynamic behavior. In the study by Avitabile et. al [8], there was excellent correlation between the data collected using the various techniques of SLDV, DIC, dynamic photogrammetry and accelerometers. Although the previous studies have shown that SLDV and DIC are both viable solutions in certain circumstances, this paper seeks to understand when to use each technique.

We present in this paper a direct and nearly simultaneous comparison between Stereo-DIC and 3D-SLDV with the goal of drawing conclusions on how best to use both techniques. A novel aspect of the research is that the comparison involved all 3 directions; both out-of-plane and in plane motion. Previous studies have focused on the out-of-plane motion solely. We show that depending on the experimental goals, one technique will be preferred. To do this we setup a modal test of a square plate to obtain modal frequencies, damping, and mode shapes in both displacement and strain. Both systems acquired the data simultaneously (at least started together as discussed later) with exactly the same shaker inputs. We present a comparison of the state-of-the-art systems (circa 2015) and discuss the pros and cons of each technique.

\section{Experimental setup}

We set up a square, corner-supported plate in the modal laboratory at Sandia National Laboratories as shown in Figure 1. The sample was a 7 inch $(177.8 \mathrm{~mm})$ square aluminum plate, $3 / 8$-inch thick $(9.5 \mathrm{~mm})$ supported by $3 / 4$-inch $(19.05 \mathrm{~mm}$ ) square posts at the four corners (see Figure 2 ) that created a complex displacement and strain field. We supported the thick bottom plate with bungee cords and excited with a shaker stinger (MB-50) applied to the back corner. An accelerometer positioned in line with the shaker stinger provided the drive point response. A Polytec 3D-SLDV system (PSV-500) measured the surface velocity and two Phantom 611 high-speed cameras nearly simultaneously imaged the surface with 50$\mathrm{mm}$ lenses $(f / 8)$ for later analysis using stereo-DIC. 


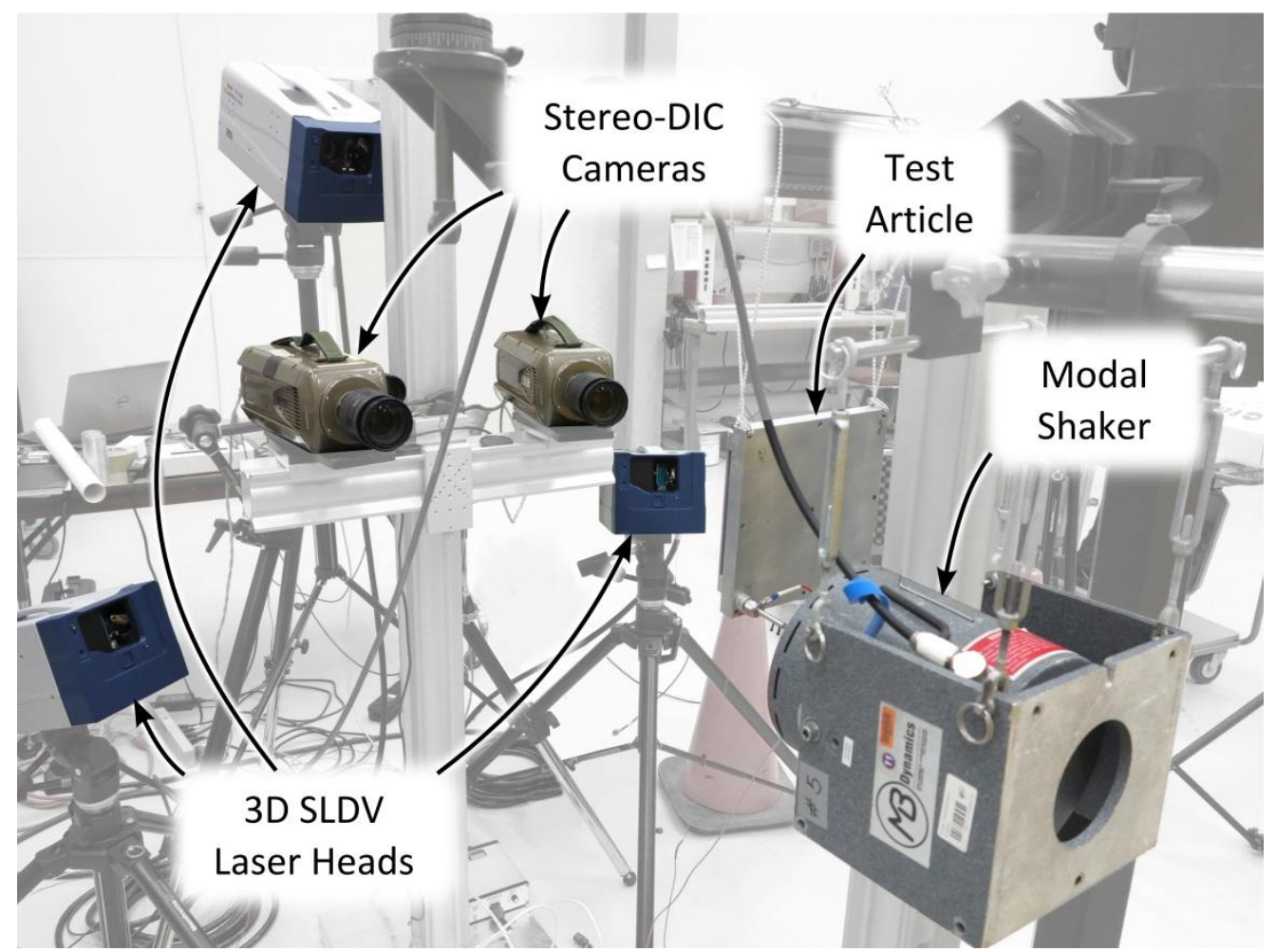

Figure 1. The experimental setup of the scanning LDV and the stereo-DIC system.

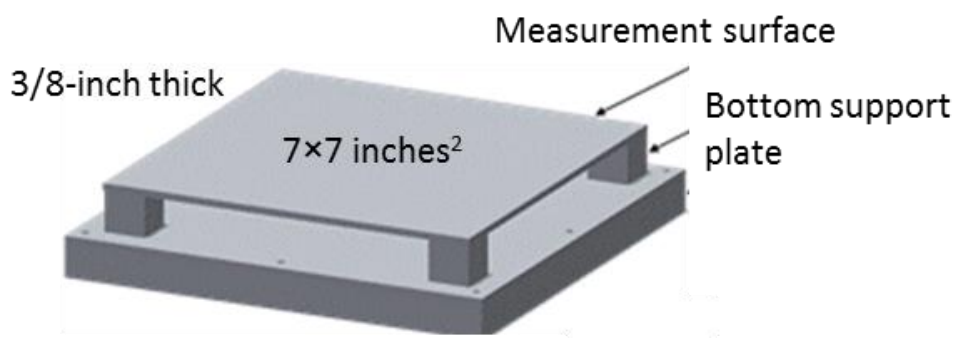

Figure 2. Square modal plate used in the experiment.

\section{DIC experimental details}

Two Phantom 611 high-speed cameras (16 GB memory option) imaged the plate during the testing. We synchronized the cameras to within the internal clock resolution of $58 \mathrm{MHz}$ using the Fsync output/input on the cameras. The cameras acquired images at $3906.25 \mathrm{~Hz}$ with an exposure of $200 \mu \mathrm{s}$ and a resolution of $800 \times 800$ pixels. We ran the cameras in 8 -bit mode (i.e. 255 counts between the white and black regions of the image) to maximize the number of frames available for the DIC modal analysis in order to allow FFT averaging for better displacement resolution. With a well exposed image there is not a need to use a higher bit depth for DIC analysis [11]. The entire camera memory was recorded for a total of 23,722 frames. Download time for all frames for both cameras over a single 1-Gigabit connection was approximately 20 minutes. Camera data was downloaded to a Phantom cine file format as a single file containing the images and other camera parameters and then broken out into 8-bit tiff 
images for analysis in the DIC software. The download rate could be cut down substantially by either using 2 computers (half the time) or a 10-Gigabit connection available on the newer cameras $(\approx 1 / 10$ the time). A USB data acquisition (DAQ) board from National Instruments (NI USB-6251) recorded the shaker input signals synchronized with the framing of the camera using a protocol supplied by Vision Research. The DAQ board recorded both the applied force and acceleration on the back of the plate. Two Nikon 24-85 mm zoom lenses set at about 50-mm imaged the plate with an aperture of $f / 8$ and a resulting magnification of $0.2 \mathrm{~mm} /$ pixel. We applied optimally sized speckles [12-14] using a base-coat of white spray paint (SEM brand self-etching primer) and a speckle coat of the same paint in black sprayed through a plastic stencil. The speckles were optimally sized to have 5 pixels/speckle to avoid aliasing while providing good spatial resolution. A laser printed calibration board with a grid of $9 \times 9$ dots and a dot spacing of 19.4-mm calibrated the stereo-camera system. We calibrated the system between the tests throughout the day to ensure we had a valid calibration for all data sets. High intensity LED light arrays (Visual Instrumentation) illuminated the object during the camera acquisition.

The time required to speckle the sample, setup the camera and DAQ system, and calibrate the cameras was approximately 2 hours. The setup time approximately matched that of the LDV including the setup of the heads and alignment of the three laser spots as discussed later.

\section{DIC Analysis}

Correlated Solutions Vic3D software (v7) analyzed the images to create the displacement data used for the modal analysis. Table 1 contains the pertinent software settings required to understand the results. We chose a large subset size relative to the underlying pattern to minimize the displacement noise and increase the precision of the correlation. This was appropriate in this case as the displacement field, even at the higher modes, was a smooth function and a linear approximation over the subset size is reasonable. We note, however, that the virtual strain gage size (VSG) in particular was very large - due mainly to the very large step size [15]. The step size, or distance between subset solution points, was driven by the need to limit the output of the DIC to $\approx 715$ points, which was easier for the modal identification software to process. The VSG could be significantly smaller and would yield less averaging in the strain field with a more reasonable subset and step size of 21 and 5 but with increased data density and DIC solution time. With higher data density the DIC data could be averaged or otherwise decimated and would likely be a better approach in the future. The DIC solution time using the settings in Table 1 was approximately 1.5 hours on a standard multi-threaded desktop PC (circa 2015). Correlated Solutions DIC software saved the data in a proprietary binary data file for which a Python script was written to read the data into MATLAB for analysis in the Sandia modal software. This step, while not required, helps simplify the data processing by not requiring that comma separated files be exported, which takes a significant period of time and increases the storage space.

The DIC data read into MATLAB were in the time domain, so the first step was to compute the FFT for each of the measurement points. For this, the time data was split into five blocks, each with 4000 samples. Displacement frequency response functions (FRFs) were computed with five averages using the $\mathrm{H} 1$ method with no windows applied to the data, since the input force was a deterministic pseudorandom signal. The accelerance FRFs were computed by differentiating the displacement data in the frequency domain twice (essentially multiplying each frequency line twice by $i \omega$, where $i$ is the complex 
variable and $\omega$ is the angular frequency of that frequency line). We discovered an issue in the frequency response functions where the phase was increasing linearly with frequency. This is believed to be due to a simple time delay between the DAQ board used to measure the drive point force and acceleration signals and the cameras due to triggering issues. A correction of $-38.7 \% \mathrm{~Hz}$ was added to the camera phases to achieve the typical behavior of a lightly damped structure where the phase is flat between modes.

Table 1. Vic3D software settings used for the analysis

\begin{tabular}{ll}
\hline Software Setting & Value \\
\hline Correlated Solutions & Vic3D v 7.2.4 (Build 393) \\
Subset & $51 \times 51$ (pixels) \\
Step Size (/data point) & 25 (pixels/data point) \\
Strain Window & 15 (data points) \\
Virtual Strain Gage & 351 (pixels) $77(\mathrm{~mm})$ \\
Pixel Size & $0.22 \mathrm{~mm} /$ pixel \\
Minimization & NSSD \\
Interpolant & $4-T a p$ \\
Image Pre-filtering & None \\
Speckle Size & $4.2 \pm 0.71$ pixels $(0.9 \mathrm{~mm})$ \\
\hline
\end{tabular}

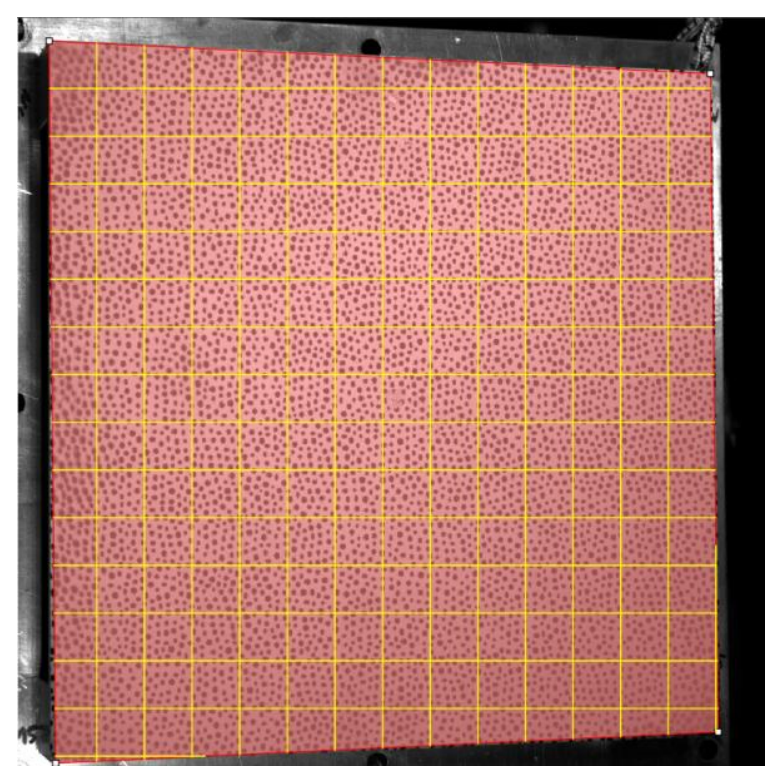

Figure 3. Overlay of the subset size on the speckle pattern over the entire plate. Note that in the solution there was an overlap of approximately $1 / 2$ the subset size.

\section{SLDV experimental details}

The 3D scanning laser Doppler vibrometer (3D SLDV) system uses three separate PSV-500 lasers to create 3D measurements. The scanning laser heads were positioned as close as possible to the manufacturer's recommendations, but compromises had to be made to accommodate the DIC cameras 
(the laser heads were placed farther from the test article and at a wider angle than would be typical for this type of test).

The laser heads were first aligned with the test article by creating a mapping between the deflections of the scanning mirrors in each laser head and an image from an external digital camera. At each point used in the alignment, we positioned the laser spot on the test article, and the corresponding point on the camera image was selected. The 400 alignment points were placed using an external script to decrease the alignment time. This allowed the laser system to interpolate between alignment points and accurately point the lasers at any point on the camera image.

The laser heads were then aligned with the test article's coordinate system. This was done by pointing the laser beams at the origin of the coordinate system, a point on the $x$ axis, a point on the $x y$ plane, and a fourth alignment point that was not coplanar with the other three. The laser software used a time-of-flight measurement from the top laser head to determine the distance of each of these points from the top head, and then computed the position and orientation of each of the laser heads based on the alignment data, which is shown in Figure 4. Accuracy for this alignment was computed by the SLDV software to be on the order of half a millimeter, which was sufficient for the 3D scan.

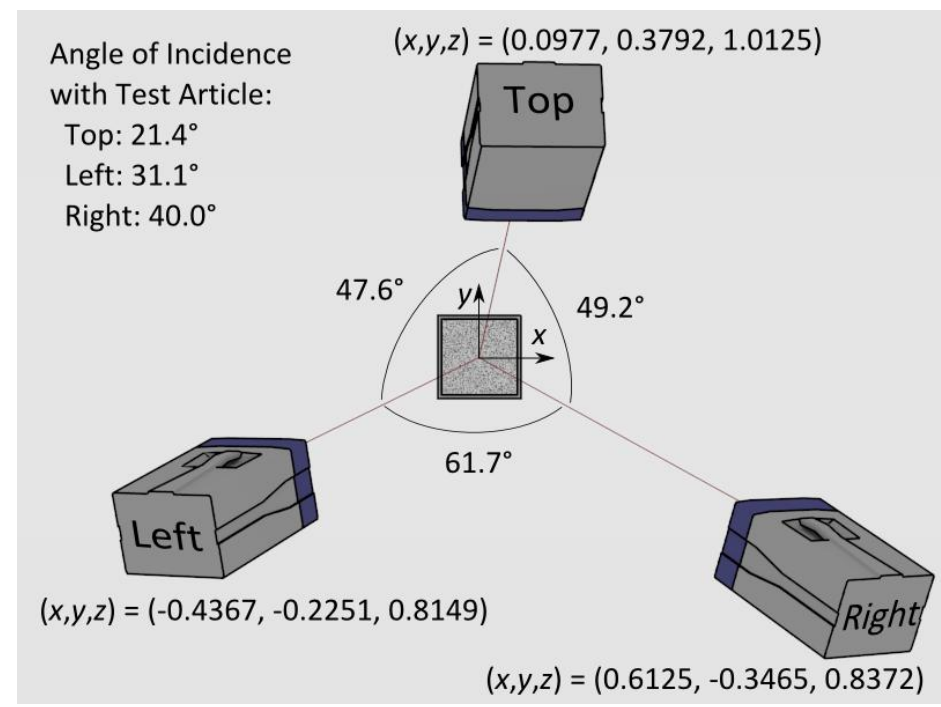

Figure 4: SLDV setup. Positions were computed from the alignment process and are shown in meters.

Figure 5 shows the measurement points defined by a grid overlaid on the camera image. The laser system then used the interpolation from the alignment with the camera image to compute mirror deflections that would point the mirrors at each point for all 3 lasers. By combining the position and orientations of the laser heads computed in the second alignment with the mirror deflections, the laser system could then triangulate the coordinates of each of the measurement points. 


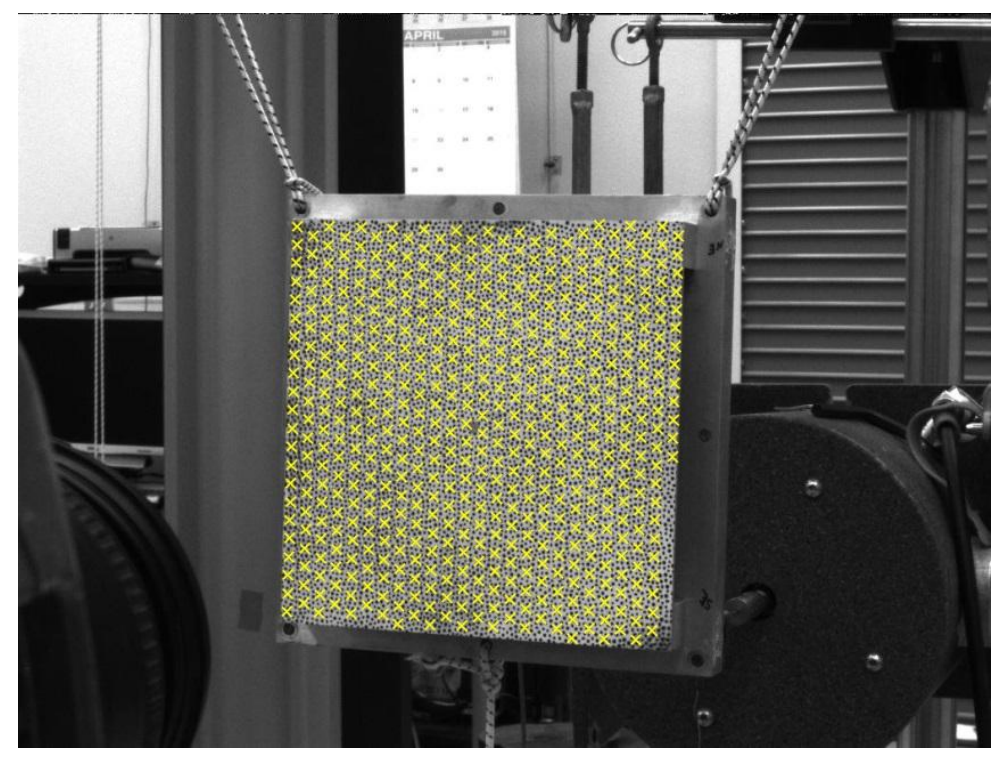

Figure 5: Measurement point locations for the 3D SLDV system.

The data acquisition system on board the SLDV system was set to measure to a frequency of $1562.5 \mathrm{~Hz}$. For each measurement, 4000 samples were taken with a sampling frequency of $3906.25 \mathrm{~Hz}$, resulting in 1600 frequency lines and a frequency resolution of $0.977 \mathrm{~Hz}$ (matching that of the DIC data). Each measurement took 1.024 seconds. Five averages were used; no windows were applied to the test data.

The Polytec DAQ system controlled the shaker via an external amplifier. A pseudorandom signal was used synchronized with the SLDV acquisition time at each measurement point. The SLDV signal generator was also used to drive the shaker for the DIC test mentioned previously. Anti-aliasing filters were used on the SLDV but were not possible for the cameras.

Frequency response functions were computed from the measured spectra using the $\mathrm{H} 1$ method. Because modal curve fitters often assume acceleration data from accelerometers, the mobility FRFs were converted to accelerance FRFs using frequency-domain differentiation. This is similar to the computation of the DIC FRFs except only a single differentiation was needed to convert from velocity to acceleration. Data were then exported to a file format that could be read by the modal curve fitter.

The scan time for the 545 SLDV points used in the modal analysis required 45 minutes. The time for 715 SLDV points to match the DIC output would be 60 minutes.

It should be remembered that the SLDV was scanning over a somewhat challenging surface with the randomly placed black dots not always returning enough signal to make a good measurement; to make the comparison fairer the SLDV test was repeated with retro-reflective tape applied to the surface of the test article. The SLDV measurement on the speckled surface was performed nearly simultaneously with the DIC measurement, so good agreement was expected between those two tests. However, to apply the retro-reflective tape, the test article was removed from its boundary conditions and detached from the shaker stinger. Because of this, small changes in the boundary conditions were expected to influence the natural frequencies and damping ratios measured for this test. 


\section{Modal analysis}

The Synthesize Modes and Correlate (SMAC) fitter [16] analyzed the input data from both the SLDV and the DIC. Figure 6 shows the modal fit for the DIC and LDV data (no retro-reflective tape). Table 2 lists the modal frequency and damping ratios calculated for the first 5 modes measured by both the SLDV and DIC - with nearly perfect agreement. This can be attributed to the test setup not being altered between the tests, ensuring any small changes due to boundary conditions or shaker effects were nonexistent. Differences in natural frequencies between the two tests were on the order of $0.02 \%$. Damping differences were higher with up to $50 \%$ difference, though extracted damping ratios are often expected to be less certain than extracted natural frequencies.

Larger differences were found after the retro-reflective tape was added, but it should be noted that these could be due to changes in boundary conditions and shaker effects due to having to take down the test article to apply the tape. The differences in natural frequency between the retro-reflective tape LDV test and the original LDV test are still only about $1 \%$. The damping was found to be higher, but it was still very lightly damped.

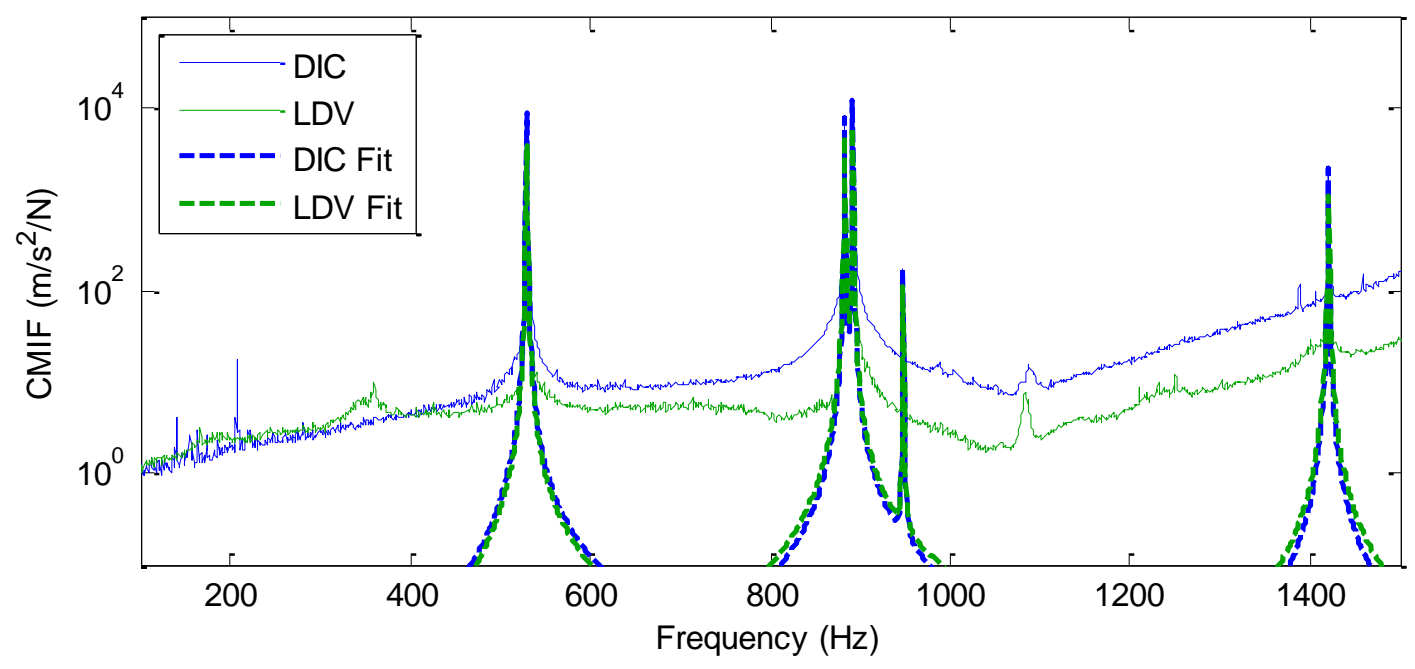

Figure 6. Complex mode indicator function showing the DIC and LDV data and the modal fits to those data. Difference in peak magnitude is largely due to the number of points used in the SVD.

Table 2. Tabulated frequency and damping of the first 5 modes.

\begin{tabular}{|c|c|c|c|c|c|c|}
\hline Mode & \multicolumn{3}{|c|}{ Frequency (Hz) } & \multicolumn{3}{c|}{ Damping (\%) } \\
\hline & DIC & LDV & LDV w/ Ret & DIC & LDV & LDV w/ Ret \\
\hline $\mathbf{1}$ & 529.4 & 529.5 & 524.8 & 0.042 & 0.042 & 0.104 \\
\hline $\mathbf{2}$ & 883.0 & 883.1 & 875.0 & 0.022 & 0.024 & 0.095 \\
\hline $\mathbf{3}$ & 891.7 & 891.7 & 883.3 & 0.015 & 0.026 & 0.098 \\
\hline
\end{tabular}




\begin{tabular}{|l|l|l|l|l|l|l|}
\hline $\mathbf{4}$ & 948.3 & 948.4 & 938.8 & 0.019 & 0.019 & 0.071 \\
\hline $\mathbf{5}$ & 1420.1 & 1420.4 & 1406.6 & 0.015 & 0.020 & 0.096 \\
\hline
\end{tabular}

Figure 7 shows the mode shapes for the first five modes calculated using the SMAC software. Each row has the same scaling for any given mode, but the scaling varies between the modes. The columns indicate different measurement approaches. Column 1 is the DIC data. Column 2 is the SLDV results, with all data points included. Column 3 is the same data as column 2, but using the Polytec software to invalidate and replace bad data points with interpolated points. Column 4 shows the SLDV data taken with the speckle pattern replaced with a retro-reflective surface to ensure the highest quality SLDV data. All modes match well in magnitude except Mode 4, where the DIC amplitudes are lower than for the SLDV. This is most likely because mode 4 has the lowest response to the input with nodes at the corner supports, and the displacement is very close to the noise floor of the DIC technique. The Modal Assurance Criterion (MAC) matrix comparing the shapes from all four approaches is shown in Table 3. The measurement points were not consistent between the DIC and SLDV tests; to compute the MAC, new shapes were created by linearly interpolating the out-of-plane component of the original shapes at a consistent set of points. 


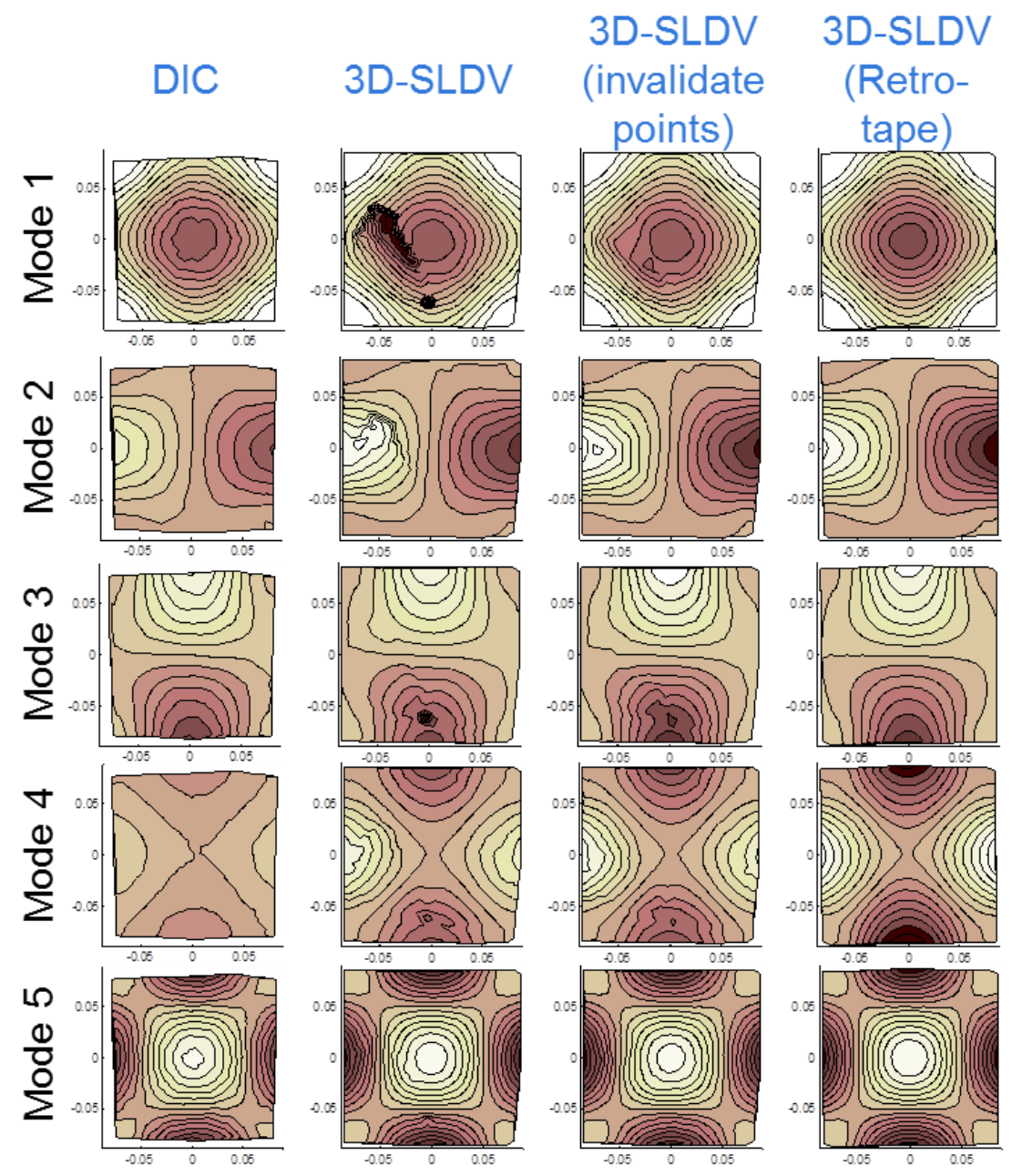

Figure 7. Mode shapes for out-of-plane motion for the DIC and different SLDV measurement approaches. 
Table 3: MAC matrix between the mode shapes shown in Figure 7.

\begin{tabular}{|c|c|c|c|c|c|c|c|c|c|c|c|c|c|c|c|c|c|c|c|c|c|}
\hline \multirow{2}{*}{\begin{tabular}{|l|} 
MAC \\
Test
\end{tabular}} & \multirow{2}{*}{\begin{tabular}{|l|} 
Test \\
Freq \\
\end{tabular}} & \multicolumn{5}{|c|}{ 3D DIC } & \multicolumn{5}{|c|}{ 3D SLDV } & \multicolumn{5}{|c|}{ 3D SLDV w/ Invalidated Pts } & \multicolumn{5}{|c|}{ 3D SLDV w/ Retrotape } \\
\hline & & & & 891.7 & & & & 883.1 & & & & & 8 & 891.9 & & 1420.3 & 524.8 & 875.0 & 883.3 & 938.8 & \\
\hline \multirow{5}{*}{$\begin{array}{l}\text { U } \\
\text { 品 }\end{array}$} & 529.4 & & & & & & & & & & & & & & & & & & & & \\
\hline & 883.0 & 000 & 000 & 000 & 007 & & 011 & 969 & 0.000 & & & & & & & & 02 & 982 & 0 & & \\
\hline & 891.7 & 000 & .000 & & 008 & 0.000 & 001 & 0.000 & & & & & & & & & 0.000 & & & & \\
\hline & 948.3 & 000 & & & 000 & & & & & & & & & & & & & & & & \\
\hline & 1420.1 & .026 & 000 & & 000 & 1.000 & 0.021 & 002 & & .000 & 980 & 0.024 & & & & 981 & 0.029 & & & & \\
\hline \multirow{5}{*}{$\begin{array}{l}\text { Dे } \\
\text { जे } \\
\text { m }\end{array}$} & 29.5 & 979 & 0.011 & 0 & 001 & 0.021 & 000 & 014 & & 204 & & 0.987 & & & & 21 & 0.983 & 002 & 0.001 & & \\
\hline & 883.1 & 000 & 59 & & 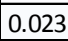 & & 014 & & & & & & & & & & 002 & & & & \\
\hline & 891.8 & 001 & 000 & & 011 & & 003 & 00 & & & & 003 & & & & & 0.001 & & & & \\
\hline & 948.4 & .001 & 003 & 001 & 0.966 & 0.000 & 0.004 & 0.020 & 0.0 & 1.000 & 0.000 & 0.001 & & & & .000 & 0.001 & & & & 00 \\
\hline & 1420.4 & 027 & 0.006 & 000 & 001 & 0.980 & 0.024 & 0.000 & & 000 & 1.0 & 0.025 & & & & 98 & 0.030 & & & & 95 \\
\hline \multirow{5}{*}{ 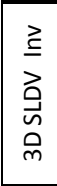 } & 29.5 & 994 & 004 & & 000 & 024 & .987 & 005 & & 001 & & 1.000 & & & & 022 & 0.998 & & & & 02 \\
\hline & 883.2 & .000 & 980 & 0.000 & 0.013 & 0.002 & 0.005 & 0.984 & 0.0 & 0.010 & .000 & 0.001 & & & & .000 & 0.000 & & & & .00 \\
\hline & 891.9 & .000 & 0 & 0.993 & 009 & 0.000 & 0.002 & 0.000 & 0.5 & & & 0.002 & & & & .000 & 0.001 & & & & 00 \\
\hline & 948.4 & 000 & 001 & & 967 & 00 & 002 & 013 & & & & 001 & & & & & 0.000 & & & & .00 \\
\hline & 1420.3 & 024 & 004 & & 0.000 & 0.981 & 0.021 & 0.000 & & .000 & & 0.022 & & & & & 0.027 & & & & .99 \\
\hline \multirow{5}{*}{ 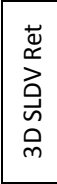 } & 24.8 & 997 & 0.002 & 000 & 000 & 0.029 & 0.983 & 0.002 & & 001 & & 0.998 & & & & & 1.000 & & & & 02 \\
\hline & 875.0 & .003 & 0.982 & 0.000 & 0.006 & 0.000 & 0.002 & 0.976 & 0.000 & 0.004 & & 0.000 & & 0.000 & & .001 & 0.000 & & 0.000 & & .00 \\
\hline & 883.3 & 000 & 000 & 0 & 010 & 0.0 & 0.001 & 01 & & 0.002 & & 001 & & & & & .000 & & & & \\
\hline & 938.8 & 0.000 & 0.008 & 0.006 & 0.957 & 0.001 & 0.000 & 0.000 & 0.009 & 0.962 & 0.001 & 0.000 & 002 & 0.007 & 971 & 0.000 & 0.000 & .008 & 0.008 & 000 & 00 \\
\hline & 1406.7 & 0.022 & 0.009 & \begin{tabular}{|l|}
0.000 \\
\end{tabular} & 0.001 & 0.978 & \begin{tabular}{|l|}
0.019 \\
\end{tabular} & 0.001 & 0.000 & 0.000 & 0.99 & 0.020 & \begin{tabular}{|l|} 
\\
\end{tabular} & 0.000 & 0.000 & 0.997 & 0.025 & 0.004 & 0.000 & 0.001 & 1.00 \\
\hline
\end{tabular}

\section{Strain Calculation}

Both the SLDV and DIC technique measure the in-plane motion and dimensions and because of this, the in-plane strains can be calculated, $\varepsilon_{x x}, \varepsilon_{y y}, \varepsilon_{x y}$. The DIC software takes the dense grid of displacement points and calculates the strain at each point using the surrounding 4 points, broken up into four triangles, similar to finite element strain calculation methods. The rigid-body motion and static out-ofplane tilt is removed and then the remaining in-plane deformation is used to calculate strains using the equations below. Because the strain will be noisy, a surrounding region of calculated strain points was spatially filtered using a decay filter. The number of data points used for averaging is called the strain window and defines the region over which the strain is averaged. A virtual strain gage (VSG) size can then be defined as:

$$
V S G=[(\text { StrainWindow }-1) * \text { StepSize }]+1
$$

A VSG size study was conducted for both the pseudo-random excitation and the dwell data. Due to the long calculation time, we chose to compare only two points with a factor of 3.5 in the VSG size. A comparison is shown in Figure 8 . The magnitudes are of the same order $\approx 4 \mu \varepsilon$, but do not match exactly. The comparison is made even more difficult by the fact that with the larger subset, the first data point is further from the edge of the sample on the right side, potentially missing the maximum strain loacation. However, due to the very small motions during the pseudorandom excitation experiment, a larger subset will often be preferred to a smaller one, due to its better ability to suppress displacement noise in the DIC results. 

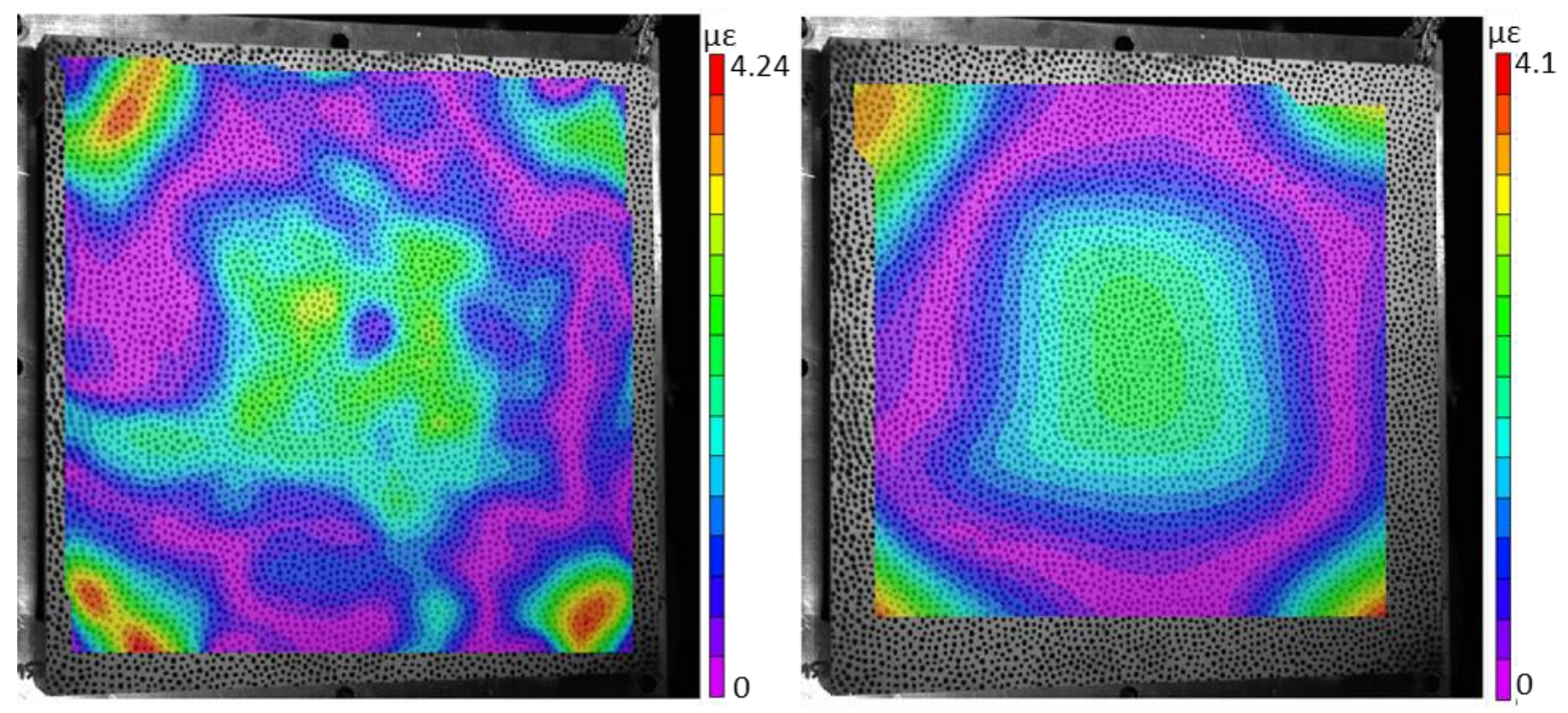

Figure 8. Comparison of e1 strain for (left) a large VSG $=351$ (Subset $=51$, Step $=25$, Strain Window $=15$ ) and (right) a small VSG $=99$ (Subset $=27$, Step $=7$, Strain Window $=15$ ).

The LDV calculates the strain by applying a user selected spatial filter to the in-plane displacement field and then calculating the infinitesimal strain between 3 neighboring data points using the following equations:

$$
\varepsilon_{x x}=\frac{\partial u}{\partial x}, \varepsilon_{x x}=\frac{\partial v}{\partial y}, \text { and } \varepsilon_{x y}=\frac{\partial u}{\partial x}+\frac{\partial v}{\partial y}
$$

Where $u$ and $v$ are the in-plane displacement in the $x$ - and $y$-directions.

To verify the strain computation of both systems for modal testing, a sine-dwell test was performed at mode 1 and mode 5. The DIC strain capability is fairly mature compared to the SLDV strain capability, so a brief study was performed to visualize how the strain shape from the SLDV changed with various levels of filtering. This study is shown in Figure 9. It was found that with a $12.5 \mathrm{~mm}$ filter, a significant amount of noise still existed in the strain shapes, so the $25 \mathrm{~mm}$ filter was used to compare to DIC data. Note that this study was done using the speckled surface of the test article (not the retro-reflective surface) so the strain data may not be as good as could be achieved. 

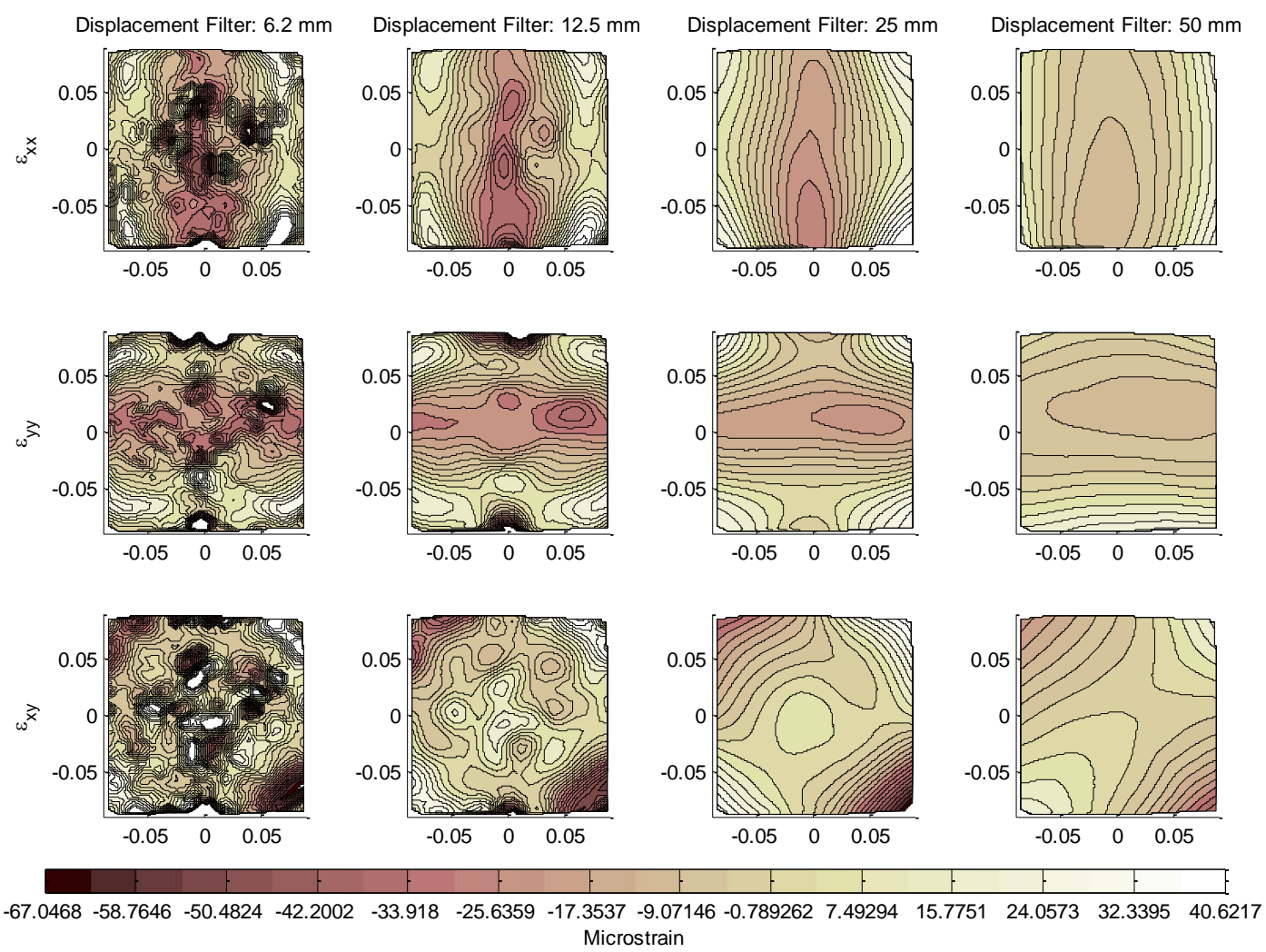

Figure 9: Computed strain from a sine-dwell test at $530 \mathrm{~Hz}$ using various filter sizes for the SLDV.

Results for the strain computation are shown in Figure 10 and Figure 11. The results are fairly consistent considering the differences between the acquisition methods and strain calculation approach. It should be noted that the $25 \mathrm{~mm}$ filter for the LDV does smooth out the noise, but it also smooths out some of the strain features that are visible in the DIC data. This is most notable in the corners of the test article near the posts. Future work will further investigate strain capabilities with the 3D SLDV system to see whether this can be improved. 
3D DIC

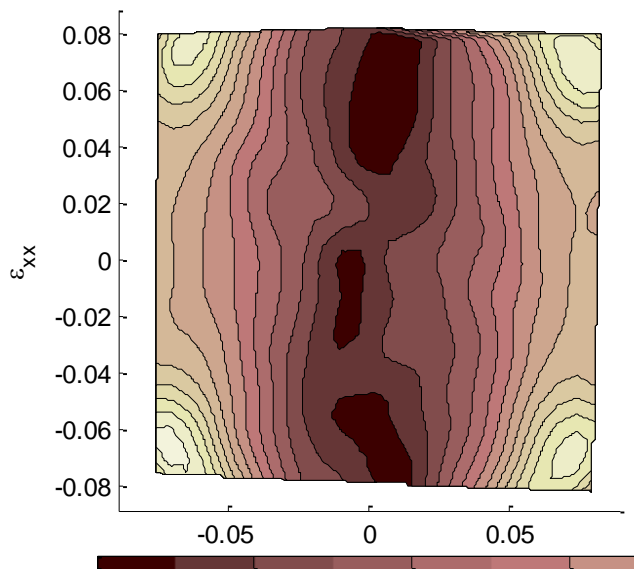

3D SLDV

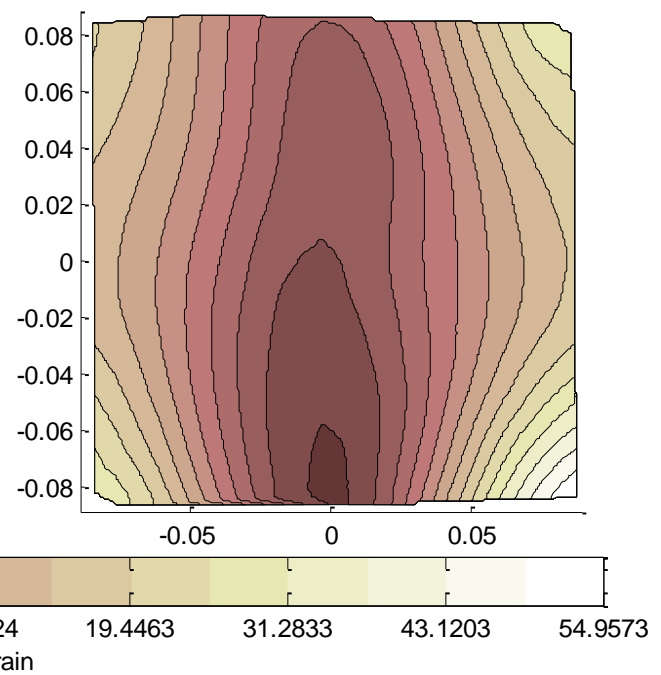

3D DIC

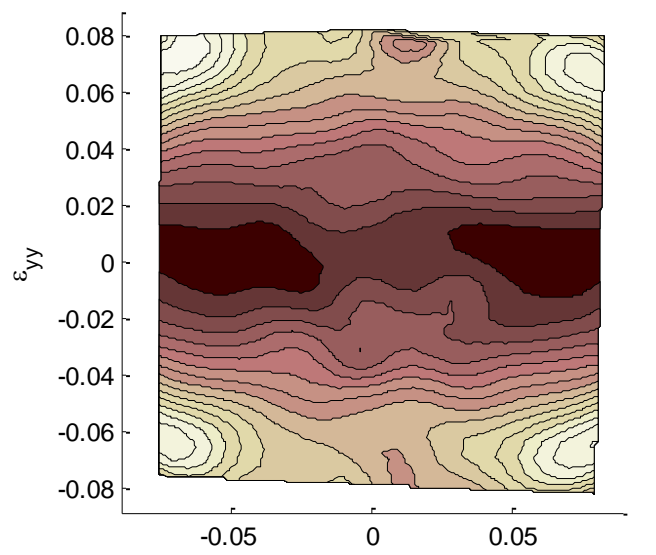

3D SLDV

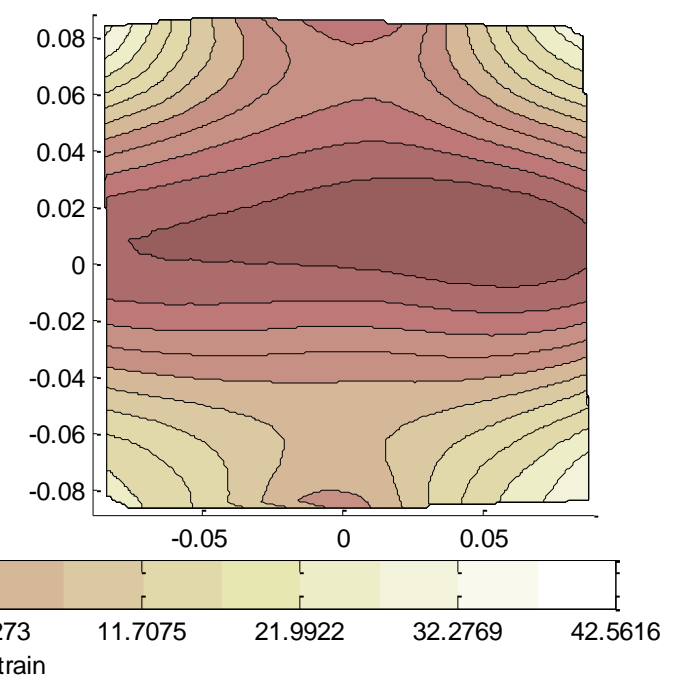




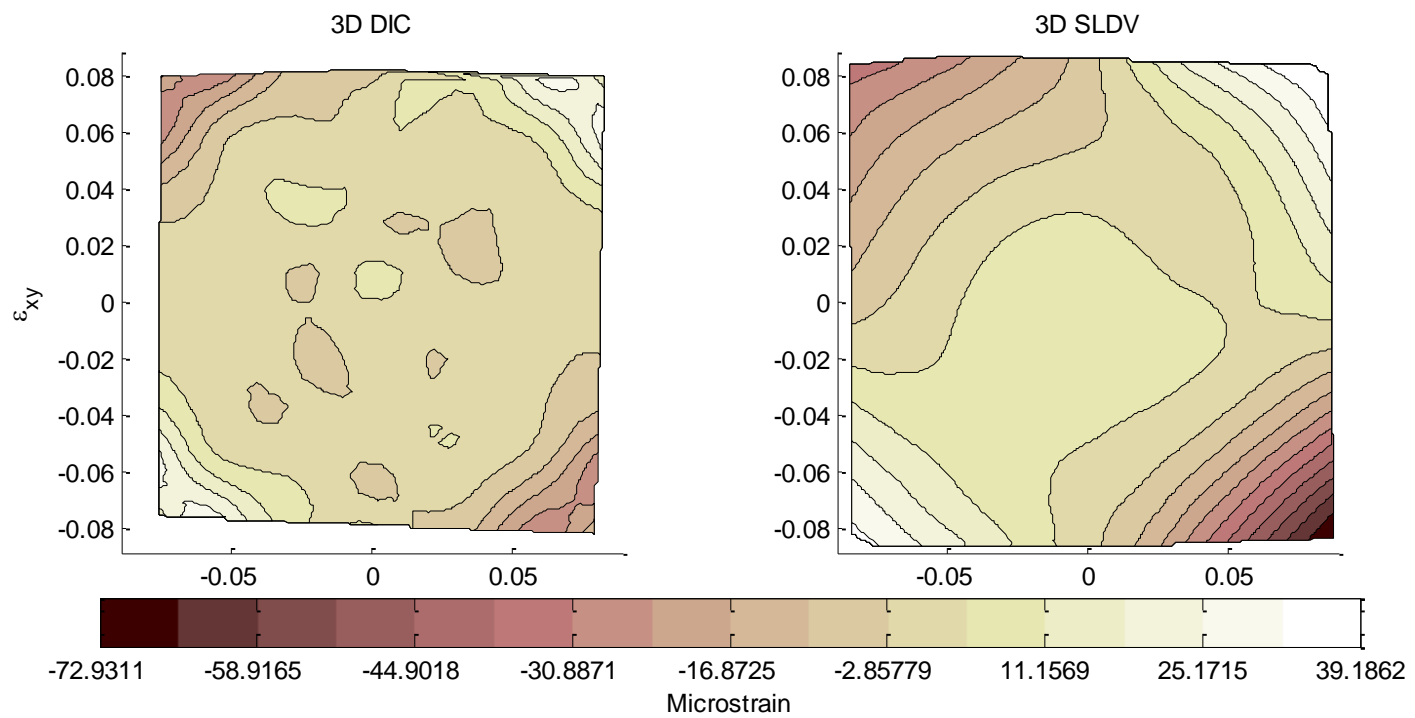

Figure 10: Strain from a sine-dwell at $530 \mathrm{~Hz}$ for both SLDV (25-mm filter) and DIC. Top is $\varepsilon_{x x}$ middle is $\varepsilon_{y y}$, and bottom is $\varepsilon_{x y}$. 
3D DIC

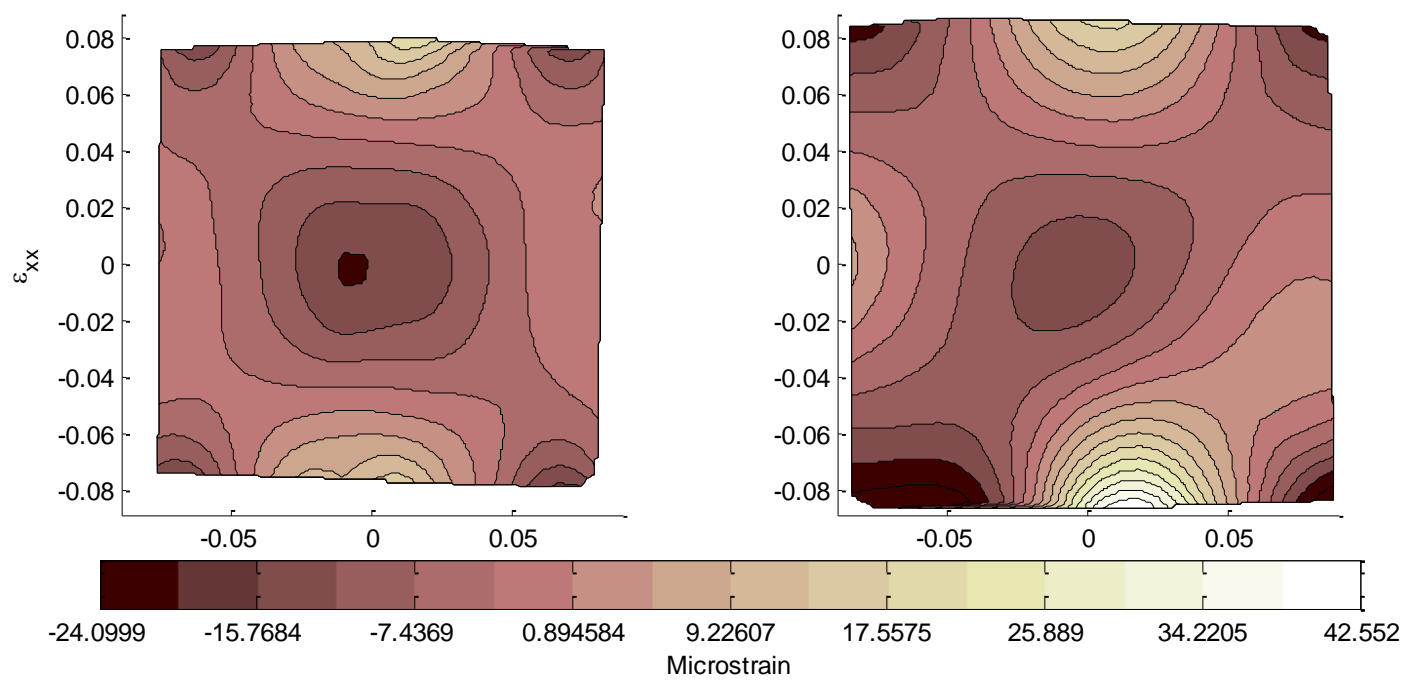

3D DIC
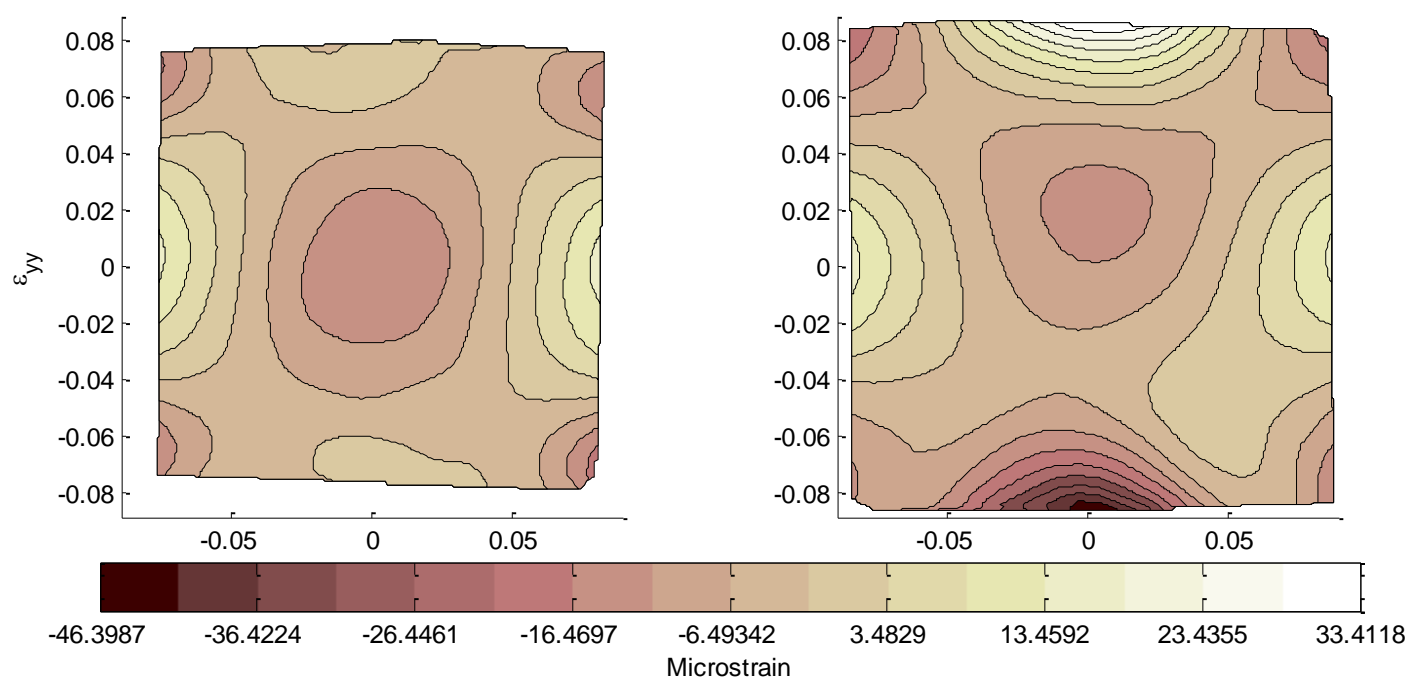

3D SLDV

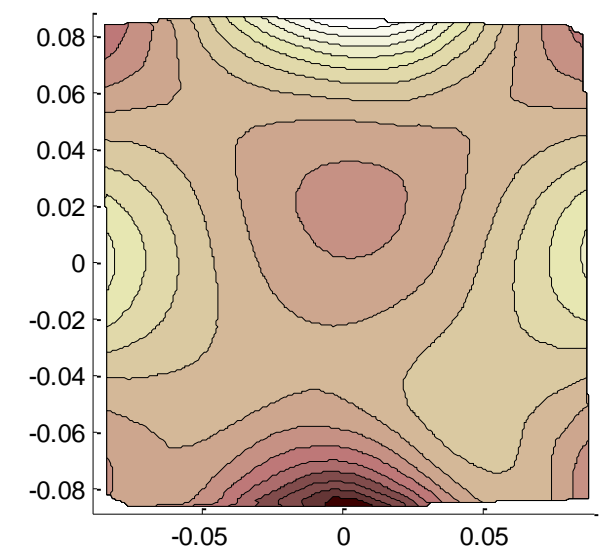

Microstrain 


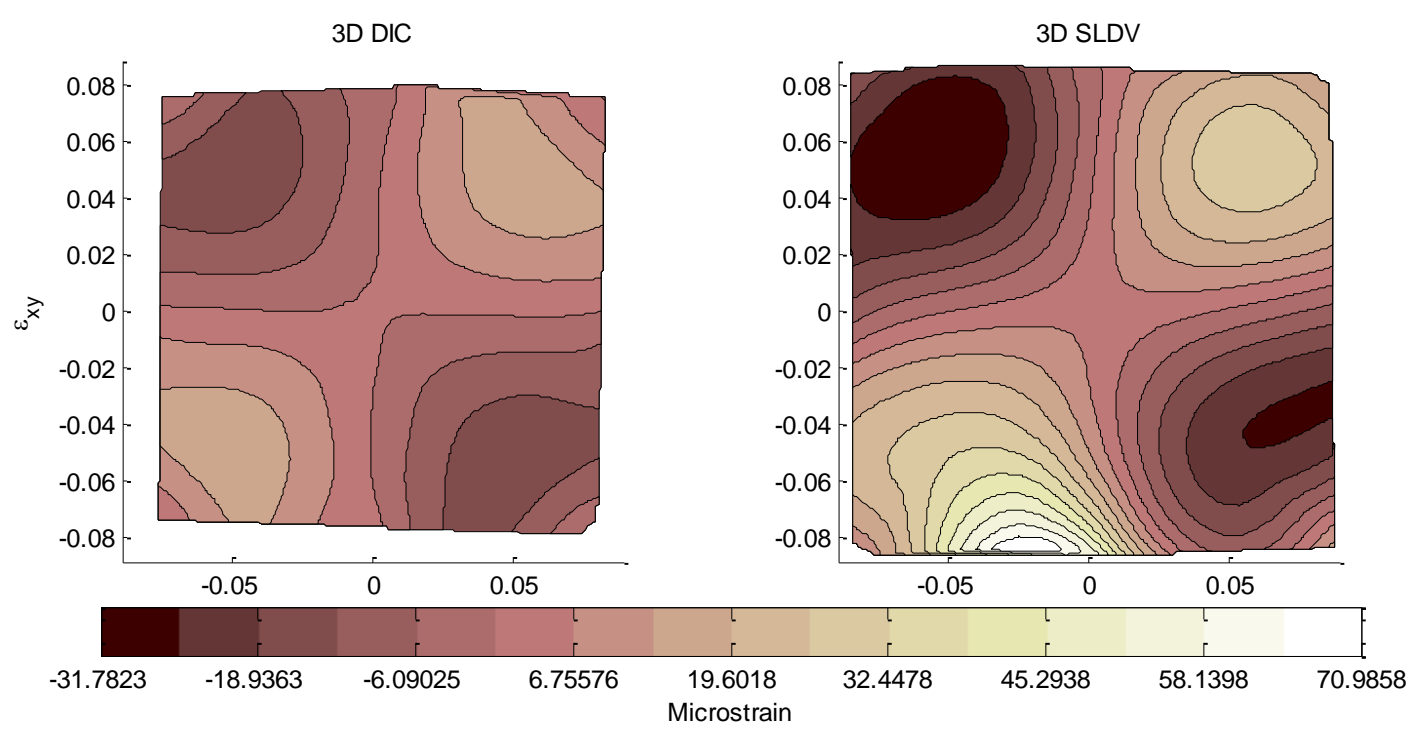

Figure 11: Strain from a sine-dwell at $1421 \mathrm{~Hz}$ for both SLDV (25-mm filter) and DIC. Top is $\varepsilon_{x x}$, middle is $\varepsilon_{y y}$, and bottom is $\varepsilon_{x y}$.

DIC has a better approach to calculating the strain for general modal and vibration testing because the speckle pattern is directly tied to the surface via the painted on pattern. The SLDV on the other hand, has the possibility of having the laser spots move during the testing, which would corrupt the results. The SLDV is therefore limited to small strain measurements where the part does not move significantly under the laser spots.

\section{Noise floor comparison between DIC and LDV}

Of fundamental importance to modal measurement is the displacement, velocity, or acceleration measurement noise floor, which will determine how much response is required for a measurement. We quantify this by looking at the noise floor of the FRF (see e.g. Figure 12). For the out-of-plane measurements $(Z)$ the clear winner is the SLDV illustrated by the much cleaner FRF. The in-plane results are approximately the same for both methods ( $X$ is illustrated but similar results occur in $Y$ ) when measuring the speckled surface, however the in-plane noise floor drops significantly when retroreflective tape is applied to the surface. This comports with the different measurement approaches used by the two techniques. The SLDV measures the velocity along the directions of the laser beams and uses a transformation to recover the components of the measurements in a global coordinate system. In this case, the beams measure primarily in the out-of-plane directions, so one would expect those measurements to be less noisy. If the transformation is poorly conditioned due to the laser beams being nearly parallel, it may amplify noise in the out-of-plane direction. However, if the laser heads are widened, they will generally have a larger angle of incidence to the part and may therefore have less laser light returning to the head which would also decrease the signal-to-noise ratio. This generally makes measurements in the in-plane direction noisier than those in the out-of-plane direction. The application of the retro-reflective tape, however, increases the laser light returned at a high angle of incidence and is shown to decrease the noise in the measurement. The exact opposite is true of the DIC, where the DIC method triangulates the out-of-plane results from the in-plane cross-correlation match. 
As the in-plane results are better aligned with the camera sensor they are more accurate than the outof-plane for DIC. In this test, the excitation was primarily out-of-plane, which will favor the LDV. More of a difference might be seen in the in-plane measurements if the excitation were in that direction.
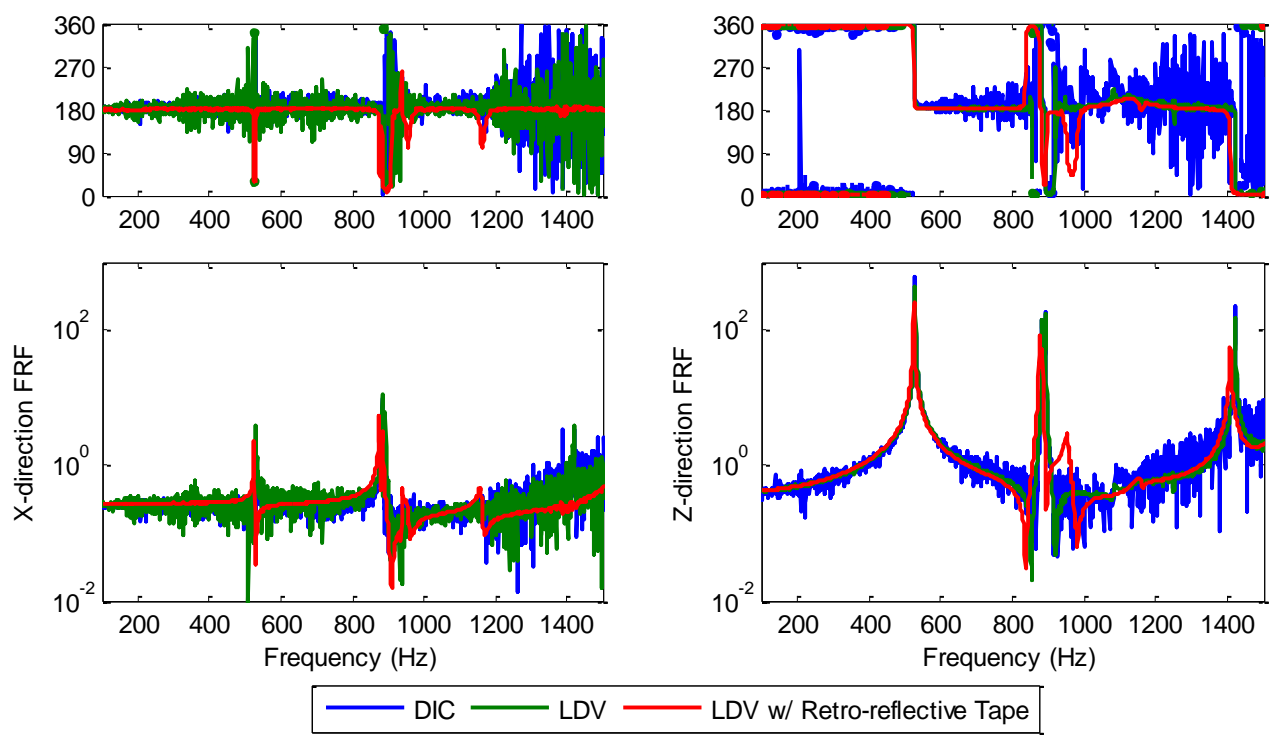

Figure 12. Noise floor comparison between DIC and LDV.

\section{Comments on DIC resolution}

A typical DIC system with the camera pixel number and FOV of this setup would typically have a displacement resolution of $\pm 2 \mu \mathrm{m}$ (or 0.01 pixels at the detector) and a strain resolution of $\pm 200 \mu \varepsilon$. The noise floor is measured by acquiring 5 to 10 static images and looking at the standard deviation of the displacements in the 3 directions. This is of course not the complete uncertainty quantification - just the estimate of the noise of the measurement. In this study, the FFT noise floor is taken as the level between the modal peaks. Figure 13 plots the FFT magnitude of the out-of-plane displacement and inplane strain from the raw DIC data. With harmonic motion, such as in modal or vibration testing, the frequency domain provides a powerful tool to better interpret the data. The horizontal red line in the figure indicates the typical time-domain displacement resolution and clearly shows that without using the data in the frequency domain, there would be no possibility of utilizing DIC for modal testing. That is, if we view this data in the time-domain only, it would appear as noise. This is because the displacement or strain noise in the temporal domain is at each data point and not distributed throughout all of the frequency bins of the FFT. When viewed in the frequency domain, at any given bin, the noise will be significantly less than for any given time-step in the raw data. We estimate the noise floor for the displacement and strain in the frequency domain from Figure 13 as $10-50$ nanometers and 0.1 to $0.02 \mu \varepsilon$ respectively and is capable of capturing the modes as illustrated in this paper. One potential pitfall for the DIC testing in the modal regime is the direct relationship between the displacement resolution and the FOV. This is most easily discussed in terms of the displacement resolution in pixels at the camera detector. With an optical magnification of $0.2 \mathrm{~mm} /$ pixel and a displacement noise floor of 10-nm, we can estimate a new frequency-domain displacement resolution at the detector of 1/10,000 of a pixel. 
Therefore if we double our field-of-view, we would need to double the noise floor to $20 \mathrm{~nm}$, or double the camera resolution to maintain a $10-\mathrm{nm}$ noise floor. High-speed cameras are typically very limited in terms of the number of pixels, so generally this will not be possible. However, larger structures will likely have proportionally larger displacements. The SLDV does not suffer from this loss of displacement resolution as the sample size increases; there are however, issues with signal strength and quality.

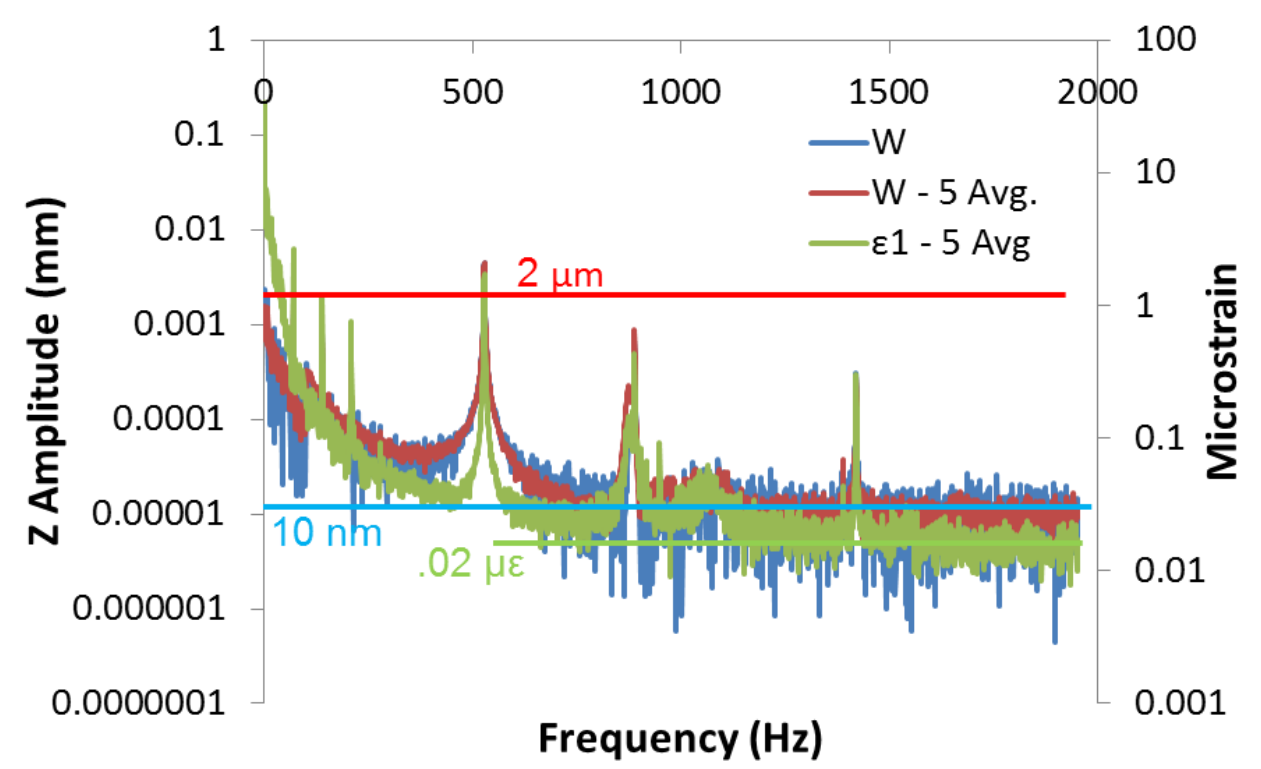

Figure 13. FFT of the Z-displacement and the in-plane strain for the plate. The red line indicates the typical displacement resolution for this FOV in a temporal experiment.

\section{Image based anti-aliasing}

A potential problem with using cameras for modal testing is temporal aliasing of the vibration response. Unlike with the SLDV and accelerometers, there are no good anti-aliasing filters for cameras. We studied a possible improvement by providing an in-plane displacement motion with a 10-second sweep between 300 and $800 \mathrm{~Hz}$. The cameras were run at $500 \mathrm{~Hz}$, which yields an aliased temporal response to the inplane displacement field. Figure 14 is the short-time Fourier transform (STFT) of a single sweep, with the frequency starting at $300 \mathrm{~Hz}$ on the left of the figure and increasing to $800 \mathrm{~Hz}$ to the right side. Note that with a Nyquist rate of $250 \mathrm{~Hz}$ we start with aliased temporal data (at $200 \mathrm{~Hz}$ decreasing) even at the beginning of the sweep. By using a shorter or longer exposure, a low-pass temporal filter can be introduced by averaging the pixel intensity over a longer time. This results in the response being blurred out at frequencies that are longer than the exposure, resulting in no measured motion. The left STFT in Figure 14 shows the results for a short exposure of $200 \mu \mathrm{s}$ and a corresponding low-pass frequency of $5000 \mathrm{~Hz}$, allowing all sweep frequencies to be aliased. The longest possible exposure (at the given frame rate) of $1900 \mu \mathrm{s}$ or $500 \mathrm{~Hz}$ is shown on the right in Figure 14. As the sweep travels from $300 \mathrm{~Hz}$ at the start of the sweep, the averaging result of the integration time of the pixel can be seen by the 
decreasing amplitude. That is, the averaging of the incoming light during the exposure time is acting as a low-pass filter with a cut-off of approximately $500 \mathrm{~Hz}$.

The authors have used one other method of antialiasing with cameras using an image intensifier and tailoring the decay time of the phosphor screen to the desired temporal low-pass filtering [17]. For example, a P-43 phosphor has a low-pass cutoff frequency of $300 \mathrm{~Hz}$ as shown in Figure 15. If the cameras imaged at $600 \mathrm{~Hz}$ with this intensifier, an effective low pass filtering would be provided by this image intensifier. Note that you can tailor the cutoff frequency by choosing different phosphors on the image intensifier. However, because image intensifiers will add noise to the image and decrease the contrast they may not be the best solution to the aliasing problem for modal testing because the image noise will increase the displacement and strain noise floor. We did not study the use of an intensifier in this experiment as we knew a priori the images were not temporally aliased. A better solution to the image intensifier would be to use a single point LDV or an accelerometer with appropriate antialiasing to ensure that the temporal bandwidth of the cameras is adequate for capturing the frequency content of the experiment. In this way, no added noise will be incurred from the intensifier.

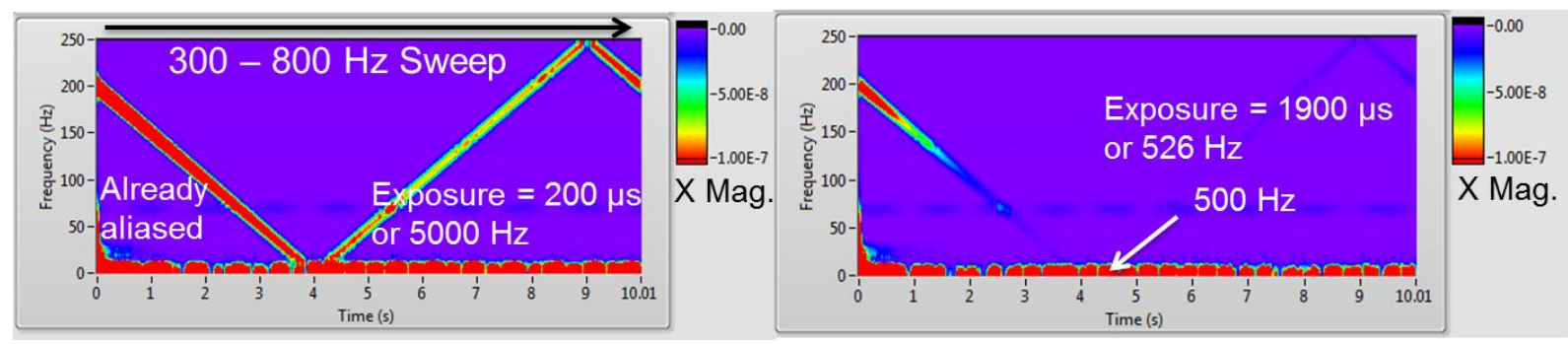

Figure 14. Short-term Fourier Transform (STFT) of the sweep data for the in-plane displacement $x$. Left figure shows a short exposure with a frame rate of $500 \mathrm{~Hz}$ and more aliased frequencies. Right figure shows the longest exposure possible and some nominal antialiasing.

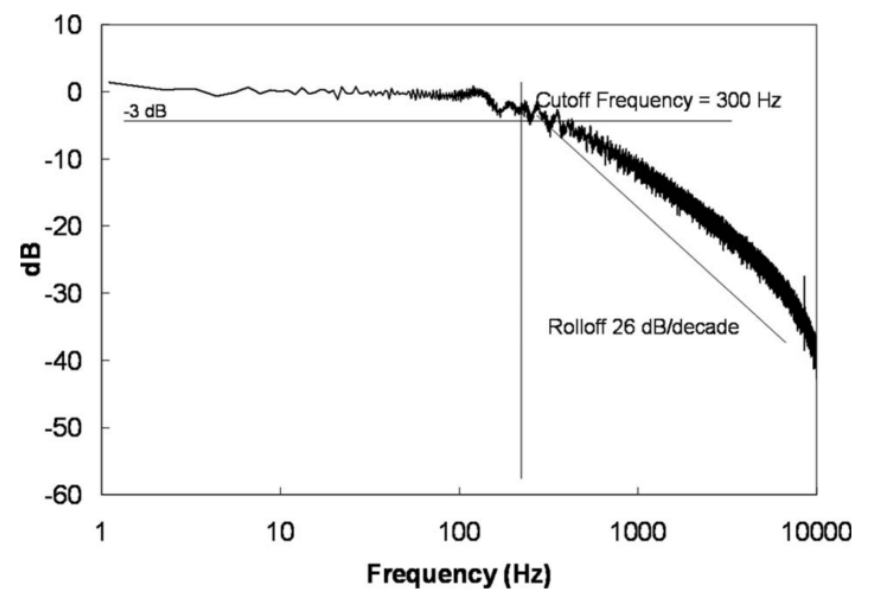

Figure 15. Low-pass temporal filtering effect of P-43 phosphor, showing a cutoff frequency of $300 \mathrm{~Hz}$, and a rolloff of $26 \mathrm{~dB} /$ decade [17]. 


\section{Conclusions}

We attempted to compare the use of SLDV and DIC for modal testing using a number of different engineering parameters. Table 4 lists these parameters, some of which are quantitative and some of which are likely to change with time (price for example), however at the point of publication the results are correct.

Table 4. Comparison between LDV and DIC.

\begin{tabular}{|l|l|l|}
\hline Comparison Metric $^{\dagger}$ & LDV & DIC \\
\hline Cost & $\approx \$ 650 \mathrm{k}$ & $\approx \$ 350 \mathrm{k}$ \\
\hline Setup time & 2 hours & 2 hours \\
\hline Acquisition Time & Hours & Seconds \\
\hline Analysis Time & Seconds & Hours \\
\hline Disp. Resolution & $\approx$ picometers & nanometers \\
\hline Strain resolution & $?$ & 5 microstrain \\
\hline Strain Calculation & Integrated - but researchy & Seamlessly Integrated \\
\hline Anti-aliasing & Included & Not possible at the moment \\
\hline Data volume & $\begin{array}{l}\text { Small (Mbytes) includes only } \\
\text { frequency data }\end{array}$ & $\begin{array}{l}\text { Large (Gbytes) but includes time } \\
\text { history }\end{array}$ \\
\hline Software & $\begin{array}{l}\text { Designed for structural } \\
\text { dynamics testing. }\end{array}$ & In its infancy. \\
\hline
\end{tabular}

The cost of two high-speed cameras and the DIC software are clearly cheaper than the full 3D scanning LDV system including software. A good argument could be made that a single scanning LDV to measure only the out-of-plane results would be adequate for a large number of situations and more comparable in price to the DIC costs where a full 3D measurement is not necessary. Furthermore, it is still unclear to the authors whether for modal testing, strain measurements are even helpful, further obviating the need for the in-plane measurements. However, for the broader topic of vibration testing, both strain and in-plane motion is likely important.

Setup time was approximately equal between the LDV and the DIC for experienced users. Both require the placement of a good amount of hardware and a calibration of the system to find the location of the cameras and measurement heads. The alignment of the 3D SLDV is slightly more involved than the calibration of the cameras. One common experimental pitfall during setup is bumping the SLDV tripod, which ruins the alignment. Moving the stereo-camera tripod is generally less of a problem for DIC.

We deemed the time to the final answer to be approximately equal. The primary difference was that the SLDV required a long time to scan the sample, while the DIC required only seconds to acquire the needed images. However, to get to the displacement results used for the modal analysis required hours for the DIC to analyze. An unattended computer can conduct both of these processes making it easy for the researcher. There may be a small benefit to DIC when testing delicate samples and it would be preferred to not have the sample under load for the full SLDV scanning time. Again, for modal testing this would be much less of an issue as a linear response to the inputs is desired and generally of small magnitudes. For vibration testing with high inputs there could be some advantages to DIC. 
The displacement noise floor for the SLDV is significantly better than the DIC results for the out-of-plane results, but approximately equal for the in-plane results. If the primary results desired are the out-ofplane motions, then the SLDV is the clear winner. However, if in-plane displacements and the calculation of strain is desired, then DIC would be the preferred solution. While it is uncertain whether strain will be useful in modal testing, for the more generic vibration testing it is likely to be important in many cases. Particularly when doing failure testing or using vibration inputs that simulate challenging environments for the structure. In these cases, the ability to quickly and accurately visualize the strain fields will be a significant advantage. This is particularly true if the sample is going to see significant motion during testing (which did not occur in this case) as the SLDV strain would become invalid as either the lasers become misaligned with each other, or end up on different regions of the test surface. Furthermore, the DIC strain methodology is more developed, better understood, and well integrated into the software. In time the SLDV may approach this.

Anti-aliasing is nearly impossible to do with cameras at this point, so the clear advantage is given to the SLDV. A possible work-around for DIC is to use a single point measurement (LDV or accelerometer) to ensure that no frequencies occur above the Nyquist rate of the cameras. This does complicate the setup and removes many of the benefits of DIC. Oversampling could work along with careful tailoring of the input vibrations or forces. This is much more difficult with modal hammer inputs, as they tend to excite a larger frequency band and roll off smoothly in the frequency domain.

Data volume for storing the results are basically equivalent. DIC will require significant "temporary" storage of the images and the DIC results, but these could be deleted after processing to limit the storage requirements and match the data that is being stored for the SLDV system. Alternately, if the time histories are going to be kept for the SLDV, storage quantities would begin to approach those needed for DIC. Depending on the research environment, the importance of this may vary from trivial to overwhelming.

Software ease of use and sophistication in the realm of modal testing are significantly better for the SLDV. The software includes input and output capabilities, anti-aliasing, and operating shape visualization that simplify modal testing. DIC software has just begun to be created for modal testing and there is room for improvement in the codes. However, for modal identification, $3^{\text {rd }}$ party codes, such as MATLAB are required that in some ways lessen the significance of the software differences.

In conclusion, depending on the type of experiment, both techniques provide a viable approach. If you have both available to you, you should tailor the measurement method to best meet the desired output data. For modal testing of structures with a hammer, the LDV system will likely provide a better approach. For vibration testing of a complex structure where strains are desired, DIC would likely be a better method. Either way, there are now viable options for full-field measurements in vibration and modal testing. 


\section{Acknowledgements}

We would like to thank Anthony Tanbakuchi for supplying the Vic data Python script and Hubert Schreier and Micah Simonsen from Correlated Solutions for many helpful conversations. Sandia is a multiprogram laboratory operated by Sandia Corporation, a Lockheed Martin Company, for the United States Department of Energy's National Nuclear Security Administration under contract No. DE-AC0494AL85000.

\section{References}

1. Beberniss, T., T. Eason, and S. Spottswood. High-speed 3D digital image correlation measurements of long-duration random vibration; recent advancements and noted limitations. in Proceedings, International Conference on Noise and Vibration Engineering (ISMA), KatholiekeUniversiteit Leuven, Belgium. 2012.

2. Helfrick, M.N., C. Niezrecki, P. Avitabile, and T. Schmidt, 3D digital image correlation methods for full-field vibration measurement. Mechanical Systems and Signal Processing, 2011. 25(3): p. 917927.

3. Niezrecki, C., P. Avitabile, C. Warren, P. Pingle, and M. Helfrick, A Review of Digital Image Correlation Applied to Structura Dynamics. AIP Conference Proceedings, 2010. 1253(1): p. 219232.

4. Schmidt, T., J. Tyson, and K. Galanulis, FULL-FIELD DYNAMIC DISPLACEMENT AND STRAIN MEASUREMENT-SPECIFIC EXAMPLES USING ADVANCED 3D IMAGE CORRELATION PHOTOGRAMMETRY: PART II. Experimental Techniques, 2003. 27(4): p. 22-26.

5. Schmidt, T., J. Tyson, and K. Galanulis, FULL-FIELD DYNAMIC DISPLACEMENT AND STRAIN MEASUREMENT USING ADVANCED 3D IMAGE CORRELATION PHOTOGRAMMETRY: PART 1. Experimental Techniques, 2003. 27(3): p. 47-50.

6. Wang, W., J.E. Mottershead, T. Siebert, and A. Pipino, Frequency response functions of shape features from full-field vibration measurements using digital image correlation. Mechanical Systems and Signal Processing, 2012. 28(0): p. 333-347.

7. Wu, P., B. Stanford, W. Bowman, A. Schwartz, and P. Ifju, DIGITAL IMAGE CORRELATION TECHNIQUES FOR FULL-FIELD DISPLACEMENT MEASUREMENTS OF MICRO AIR VEHICLE FLAPPING WINGS. Experimental Techniques, 2009. 33(6): p. 53-58.

8. Avitabile, P., C. Niezrecki, M. Helfrick, C. Warren, and P. Pingle, Noncontact measurement. Techniques for model correlation. Sound and Vibration, 2010. 44(1): p. 8.

9. Warren, C., C. Niezrecki, P. Avitabile, and P. Pingle, Comparison of FRF measurements and mode shapes determined using optically image based, laser, and accelerometer measurements. Mechanical Systems and Signal Processing, 2011. 25(6): p. 2191-2202.

10. Ehrhardt, D., S. Yang, T. Beberniss, and M. Allen, Mode Shape Comparison Using ContinuousScan Laser Doppler Vibrometry and High Speed 3D Digital Image Correlation, in Special Topics in Structural Dynamics, Volume 6, G. Foss and C. Niezrecki, Editors. 2014, Springer International Publishing. p. 321-331.

11. Reu, P., All about Speckles: Contrast. Experimental Techniques, 2015. 39(1): p. 1-2.

12. Sutton, D.A., J.J. Orteu, and H.W. Schreier, Image Correlation for Shape, Motion and Deformation Measurements. 2009, New York, NY: Springer.

13. Reu, P., All about Speckles: Aliasing. Experimental Techniques, 2014. 38(5): p. 1-3.

14. Reu, P., All about Speckles: Speckle Density. Experimental Techniques, 2015. 39(3): p. 1-2.

15. Reu, P., Virtual Strain Gage Size Study. Experimental Techniques, 2015. 39(5): p. 1-3. 
16. Hensley, D.P. and R.L. Mayes. Extending SMAC to multiple reference FRFs. in Proceedings of the 24th International Modal Analysis Conference (IMAC XXIV), St. Louis, MI. 2006.

17. Reu, P.L. and B.D. Hansche, Optical temporal frequency low-pass filtering and heterodyning with a microchannel plate. Optical Engineering, 2008. 47(7). 